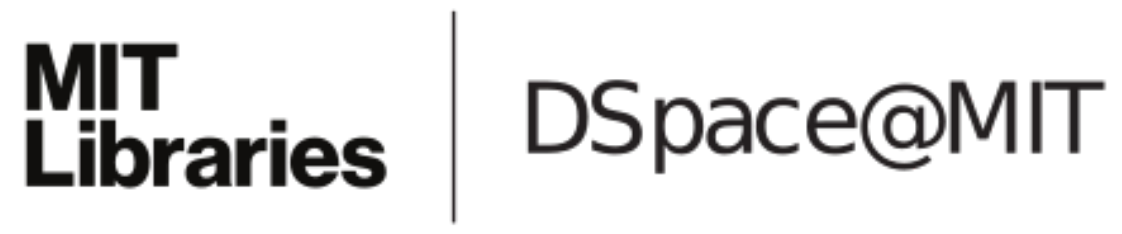

\author{
MIT Open Access Articles
}

\begin{abstract}
Dynamic susceptibility of supercooled water and its relation to the dynamic crossover phenomenon
\end{abstract}

The MIT Faculty has made this article openly available. Please share how this access benefits you. Your story matters.

Citation: Zhang, Yang et al. "Dynamic susceptibility of supercooled water and its relation to the dynamic crossover phenomenon." Physical Review E 79.4 (2009): 040201. (C) 2010 The American Physical Society.

As Published: http://dx.doi.org/10.1103/PhysRevE.79.040201

Publisher: American Physical Society

Persistent URL: http://hdl.handle.net/1721.1/51006

Version: Final published version: final published article, as it appeared in a journal, conference proceedings, or other formally published context

Terms of Use: Article is made available in accordance with the publisher's policy and may be subject to US copyright law. Please refer to the publisher's site for terms of use. 


\title{
Dynamic susceptibility of supercooled water and its relation to the dynamic crossover phenomenon
}

\author{
Yang Zhang, ${ }^{1}$ Marco Lagi, ${ }^{1,2}$ Emiliano Fratini, ${ }^{2}$ Piero Baglioni, ${ }^{2}$ Eugene Mamontov, ${ }^{3}$ and Sow-Hsin Chen ${ }^{1, *}$ \\ ${ }^{1}$ Department of Nuclear Science and Engineering, Massachusetts Institute of Technology, Cambridge, Massachusetts 02139, USA \\ ${ }^{2}$ Department of Chemistry and CSGI, University of Florence, Florence I 50019, Italy \\ ${ }^{3}$ Spallation Neutron Source, Oak Ridge National Laboratory, Oak Ridge, Tennessee 37831, USA
}

(Received 15 January 2009; published 7 April 2009)

\begin{abstract}
We study the dynamic susceptibility $\chi_{T}(Q, t)$ of deeply supercooled water by means of quasielastic neutron scattering and molecular dynamics simulations. Both techniques show an increase in the peak height of $\chi_{T}(Q, t)$ as the temperature is lowered toward the dynamic crossover temperature $T_{L}$. Below $T_{L}$, the peak height decreases steadily. We attribute this phenomenon to the change in slope of the Arrhenius plot of the translational relaxation time at $T_{L}$. In contrast, the peak height of the calculated four-point correlation function $\chi_{4}(Q, t)$, directly related to the size of dynamic heterogeneity, increases toward and below $T_{L}$.
\end{abstract}

DOI: 10.1103/PhysRevE.79.040201

PACS number(s): 61.05.fg, 64.70.qj, 66.30.jj

The counterintuitive properties of supercooled water have been fascinating scientists for the past 40 years [1,2]. The difficulty in investigating these properties experimentally below $T_{H}=235 \mathrm{~K}$ (homogeneous nucleation temperature) has been overcome over the years using different kinds of confinement that prevent crystallization into hexagonal ice [2-5]. Nevertheless, the structure and dynamics of deeply supercooled water remain a subject of frequent debates among the scientific community.

Much theoretical [6-8], experimental [2-5,9], and numerical evidence $[10,11]$ suggests that some definite change in behavior takes place in the thermodynamic and transport properties of supercooled water at and around $T_{L}$ $=225 \pm 5 \mathrm{~K}$. At ambient pressure, bulk liquid water shows an anomalous increase in thermodynamic response functions and an apparent divergent behavior of transport properties, on approaching an apparent singular temperature $T_{L}$. When confined water is deeply supercooled, both the Arrhenius plots of the experimentally measured translational relaxation time and the inverse of the diffusion constant show a switch from a super-Arrhenius behavior (at high temperatures) to an Arrhenius behavior (at low temperatures) across $T_{L}$. This socalled dynamic crossover phenomenon is also visible as a peak in the temperature dependence of many thermodynamic response functions, such as the specific heat $[5,12]$ and the thermal expansion coefficient $[13,14]$. The similar crossover in hydration water of proteins seems to have an important effect on the low-temperature dynamics of the protein itself $[4,15]$.

In this Rapid Communication, we present how another dynamic quantity can be used to identify the crossover temperature-the dynamic susceptibility $\chi_{T}(Q, t) . \chi_{T}(Q, t)$ is one of the dynamic response functions and it is defined as the derivative of the self-density correlation function $F_{S}(Q, t)$ with respect to the temperature $T$, namely,

$$
\chi_{T}(Q, t)=-\left(\frac{\partial F_{S}(Q, t)}{\partial T}\right)_{P} .
$$

\footnotetext{
*Author to whom correspondence should be addressed. sowhsin@mit.edu
}

$\chi_{T}(Q, t)$ is not only a measure of the temperature-induced dynamic fluctuations, but also an experimentally accessible quantity related to a genuine multipoint correlator that identifies the dynamic heterogeneity-the four-point correlation function $\chi_{4}(Q, t)[16] . \chi_{4}(Q, t)$ has a peak when plotted as a function of time, the position of which indicates the characteristic relaxation time of the system, and the height of which is related to the volume where correlated motions take place. Experimentally, $\chi_{T}(Q, t)$ is a much easier quantity to measure than $\chi_{4}(Q, t)$, which quantifies the amplitude of the spontaneous fluctuations. However, the two susceptibilities are related to each other by the fluctuation-dissipation theorem $[16,17]$.

In this Rapid Communication, we show both by experiments [quasielastic neutron scattering (QENS)] and by molecular-dynamics (MD) simulations that the peak height of $\chi_{T}(Q, t)$ for supercooled water increases as $T$ approaches $T_{L}$, but below $T_{L}$ the peak height decreases. In order to deeply supercool water we confined it in cement paste with a water/dry powder mass ratio equal to 0.4 . Differential scanning calorimetry (DSC) measurements and near-infrared (NIR) spectra confirm that after eight days all the water in the sample is confined into the developing calcium silicate hydrate (C-S-H) gel [18], and the NIR crystallization peak (due to formation of hexagonal ice) disappears $[5,19]$. The high-resolution backscattering spectrometer BASIS at Spallation Neutron Source (SNS) in Oak Ridge National Laboratory (ORNL) was then used to measure the broadening of the elastic peak of the hydrogen atom dynamic structure factor $S_{H}(Q, E)$. Using incident neutrons of $2.08 \mathrm{meV}$, BASIS is capable of measuring a dynamic range as large as $\pm 100 \mu \mathrm{eV}$ with an elastic energy resolution of $3 \mu \mathrm{eV}$ [full width at half maximum (FWHM)]. In this experiment, both an eight-day-old $\mathrm{H}_{2} \mathrm{O}$ hydrated sample and a dry sample of cement powder were measured. The scattering from the dry sample was rather small due to the lack of hydrogen atoms and thus was subtracted out as a background from the wet sample.

The measured QENS spectrum was analyzed with the relaxing cage model (RCM) [20], which has been tested extensively by MD simulations [20,21] and QENS experiments $[3-5,21]$. According to this model, the measured spectral intensity distribution is expressed as 


$$
I(Q, E)=N\left\{f(Q) \delta(E)+[1-f(Q)] F T\left[F_{S}(Q, t)\right]\right\} \otimes R(Q, E),
$$

where $N$ is the normalization factor and $f(Q)$ is the elasticscattering component that takes into account the contribution coming from hydrogen atoms that cannot migrate more than a distance $\sim 2 \pi / Q$, within the experimental observation time window. $F_{S}(Q, t)$ is the self-intermediate scattering function (SISF) of the hydrogen atoms in the hydration water,

$$
F_{S}(Q, t)=\left\langle F_{l}(Q, t)\right\rangle=\left\langle\exp \left\{i \mathbf{Q} \cdot\left[\mathbf{r}_{l}(t)-\mathbf{r}_{l}(0)\right]\right\}\right\rangle,
$$

where $R(Q, E)$ is the $Q$-dependent energy resolution function as obtained by a low-temperature run at $10 \mathrm{~K}$ using the hydrated sample.

A sum of four Gaussian functions was used to represent the $R(Q, E)$. Generally, the SISF is a product of the translational part $F_{T}(Q, t)$ and the rotational part $F_{R}(Q, t)$, i.e., $F_{S}(Q, t)=F_{T}(Q, t) F_{R}(Q, t)$. By using only the spectra with $Q<1 \AA^{-1}$, the rotational contribution can be made negligibly small [20]. So the SISF is modeled as follows:

$$
F_{S}(Q, t) \approx F_{T}(Q, t)=F^{v}(Q, t) \exp \left[-\left(\frac{t}{\tau(Q, T)}\right)^{\beta}\right],
$$

where the first factor $F^{v}(Q, t)$ represents the short-time vibrational motion of the water molecules in the cage. This factor affects the SISF in sub-ps time scale and thus is not an observable effect in this experiment [i.e., $F^{v}(Q, t) \sim 1$ ].

The second factor, the translational relaxation term, contains the stretch exponent $\beta$ and the $Q$-dependent translational relaxation time $\tau(Q, T)$, which is a strong function of temperature. $\tau(Q, T)$ follows a power-law $Q$ dependence as $\tau(Q, T)=\tau_{0}(T)(a Q)^{-\gamma}, \quad$ where $\quad a=0.5 \AA \quad[20] . \quad$ The $Q$-independent average translational relaxation time $\langle\tau\rangle$ is then evaluated as $\langle\tau\rangle=\left(\tau_{0} / \beta\right) \Gamma(1 / \beta)$, where $\Gamma$ is the gamma function. It essentially gives a measure of the structural relaxation time of the hydrogen-bond cage surrounding a water molecule. The QENS spectra for each temperature were analyzed for four $Q$ values simultaneously $\left(0.3,0.5,0.7,0.9 \AA^{-1}\right)$ to extract $\tau_{0}(T)$ and $\beta$ and consequently to evaluate the average translational relaxation time $\langle\tau\rangle$.

It is debatable whether the relaxation process we observed with QENS in supercooled confined water is the slowest relaxation of the system ( $\alpha$ relaxation) [22-26]. Nevertheless, it is verified by both experiments (Fig. 1) and MD simulations [Fig. 3(a)] that the relaxation process we observed obeys a stretched exponential decay. Since this is the only a priori assumption of the relaxing cage model, our results are still interesting to report even if the measured process is not the genuine $\alpha$ process: the observation of a crossover is anyway a feature that is revealing some changes in the structure of the hydrogen bond network.

Figure 1 shows two spectra taken above and below the crossover temperature. One should note from these two plots that the wing of the spectra is significantly larger than the resolution function. This is because the Fourier transform of the stretched exponential form of SISF produces a significantly wide skirt and allows meaningful data analyses.

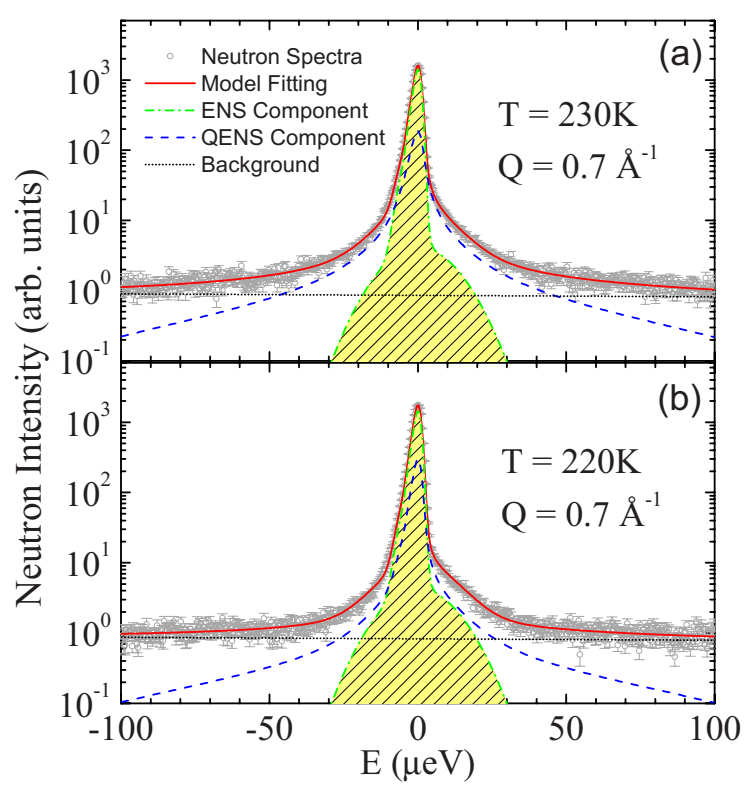

FIG. 1. (Color online) Illustration of the analysis of QENS spectra at two typical temperatures, (a) $230 \mathrm{~K}$ and (b) $220 \mathrm{~K}$ at $Q=0.7 \AA^{-1}$. The hollow circles are the measured neutron intensity as a function of the energy transfer $E$. The solid line is the fitted curve using the RCM model. The dashed-dotted line with shadow is the elastic-scattering component, whose asymmetric shape derives from the asymmetric $Q$-dependent resolution function. The dashed line is the quasielastic scattering component. The dotted line is the background.

Figure 2(a) shows the experimentally extracted $\langle\tau\rangle$ as a function of $1 / T$. A gradual transition (change in slope) from a super-Arrhenius at high temperatures (nonlinear behavior) to an Arrhenius behavior at low temperatures (linear behavior) can be seen as the temperature is cooled down across $T_{L}$. To determine the dynamic crossover temperature $T_{L}$ experimentally, we plotted the derivative of the Arrhenius plot, $d \ln \langle\tau\rangle / d(1 / T)$ [Fig. 2(b)]. The peak, or equivalently the change in the slope in the Arrhenius plot, suggests that $T_{L}=225 \pm 5 \mathrm{~K}$, in agreement with the peak position of the DSC data reported in Fig. 2(d) $\left(T_{L}=227 \pm 2 \mathrm{~K}\right)$.

We are now going to show that it is possible to evaluate this crossover temperature also from the dynamic response function $\chi_{T}(Q, t)$. Figure 2(c) shows the dynamic response function for several temperatures as a function of time. $\chi_{T}(Q, t)$ is calculated using finite differences.

The peak height of $\chi_{T}(Q, t), \chi_{T}^{*}(Q)$, grows as $T$ is lowered and reaches a maximum at $T_{L}=227 \pm 5 \mathrm{~K}$, but this growth is interrupted when the dynamic crossover sets in. The reason for this behavior is clear if one considers that, combining Eqs. (1) and (4),

$\chi_{T}(Q, t)=-\frac{\partial F_{S}(Q, t)}{\partial T}=F_{S}(Q, t) \beta\left(\frac{t}{\tau(Q, T)}\right)^{\beta} \frac{\partial \ln \tau(Q, T)}{\partial(1 / T)} T^{-2}$.

The only parameter in $F_{S}(Q, t)$ that has to be differentiated with respect to $T$ is $\tau(Q, T)$ since $\beta$ remains almost constant and close to $0.5 \pm 0.1$ as $T$ is lowered [27]. $\chi_{T}^{*}(Q)$ is therefore 

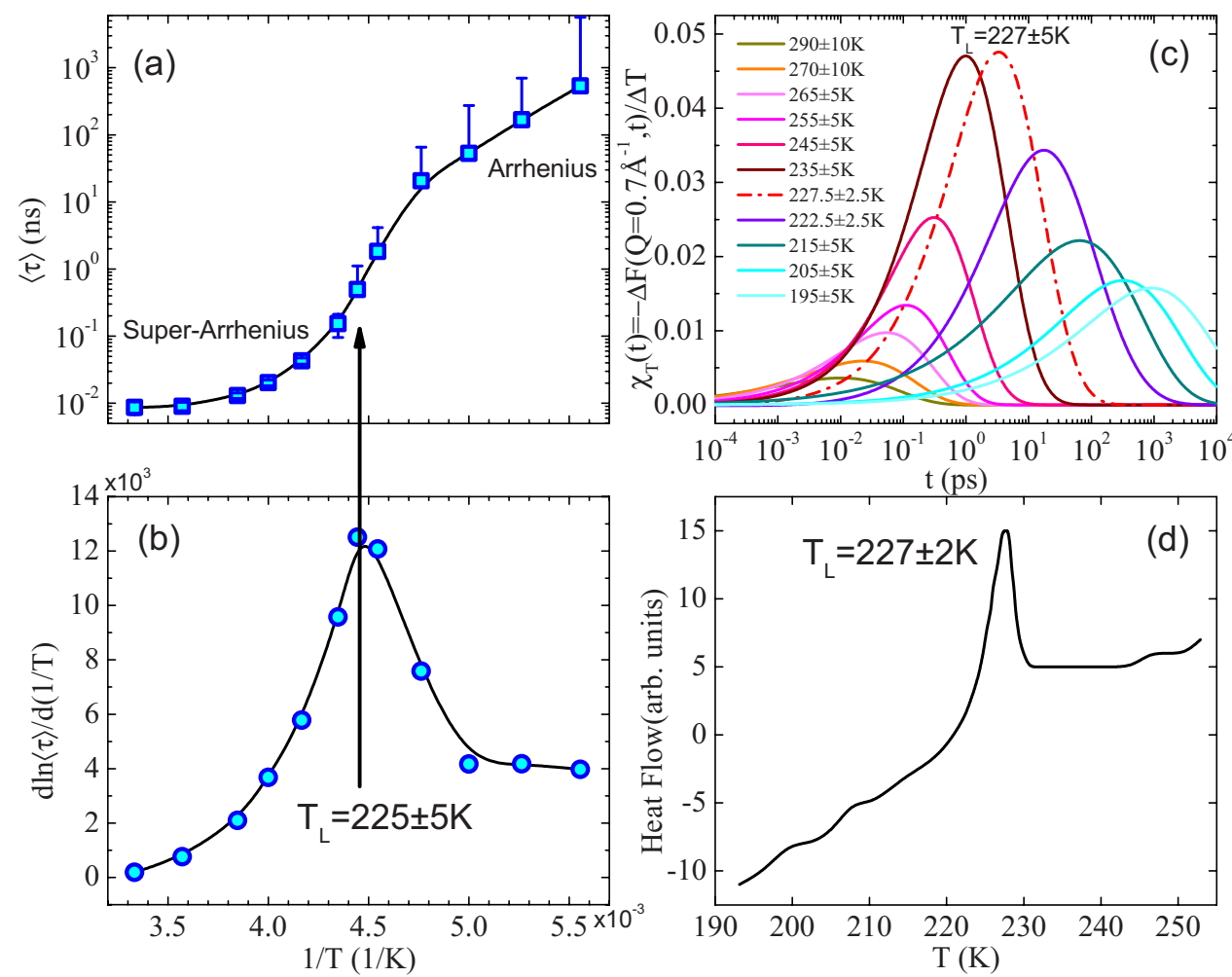

FIG. 2. (Color online) (a) Arrhenius plot of the experimental translational relaxation time for water confined in aged cement paste. (b) Derivative of the Arrhenius plot of the translational relaxation time. (c) Dynamic response function calculated with finite differences of the SISF. (d) DSC curve of water confined in eight-day-old hydrated cement paste.

directly proportional to the change in slope of the Arrhenius plot of $\tau(Q)$ [Fig. 2(b)].

Therefore, the three quantities $\chi_{T}(Q, t),\langle\tau\rangle$, and DSC heat flow all agree in evaluating the crossover temperature as $T_{L} \sim 225 \pm 5 \mathrm{~K}$. These experimental findings demonstrate that there are well-defined thermodynamic and dynamic signatures in the response functions of the existence of the crossover temperature $T_{L}$.

To make sure that these phenomena are inherent properties of water and not due to the confinement, we ran a simulation of a model bulk water, four-point transferable intermolecular potential modified for the Ewald sums (TIP4P-Ew) [28]. The dynamic crossover in the Arrhenius plot of the self-diffusion constant has been previously observed with simulations of bulk water using other water models [10,29].

We calculated long MD trajectories for a box of 512 water molecules of up to $1 \mu$ s in the NVT ensemble. The systems were considered equilibrated when the mean-square displacement of the water molecules was larger than $0.1 \mathrm{~nm}^{2}$ [30] [see Fig. 3(c)]. We then calculated the SISF for the oxygen atoms for five $Q$ values $\left(0.4,0.5,0.6,0.7,0.8 \AA^{-1}\right)$ and fit the data according to the RCM described above [see Fig. 3(a)]. Figures 3(b) and 3(d) show the Arrhenius plots of the transport properties obtained from the trajectories: the translational relaxation time $\langle\tau\rangle$ and the inverse of the selfdiffusion constant $1 / D$, respectively. Both the plots show a dynamic crossover at $T_{L}=215 \pm 5 \mathrm{~K}$, analogous to the one in Fig. 2(a). As a side note, $\left\langle\tau\left(T_{L}\right)\right\rangle$ is between 1 and $10 \mathrm{~ns}$ range for both experiments and simulations, confirming the general behavior of many glass formers [31].
The upper panel of Fig. 4 shows the dynamic response function $\chi_{T}(Q, t)$ extracted from the trajectories. Error bars on $\chi_{T}(Q, t)$ are on the order of $10^{-2} \mathrm{~K}^{-1}$. As also observed experimentally, $\chi_{T}^{*}(Q)$ decreases after the dynamic crossover temperature $T_{L}=215 \mathrm{~K}$.

The same phenomenon is not observed for $\chi_{4}(Q, t)$ calcu-

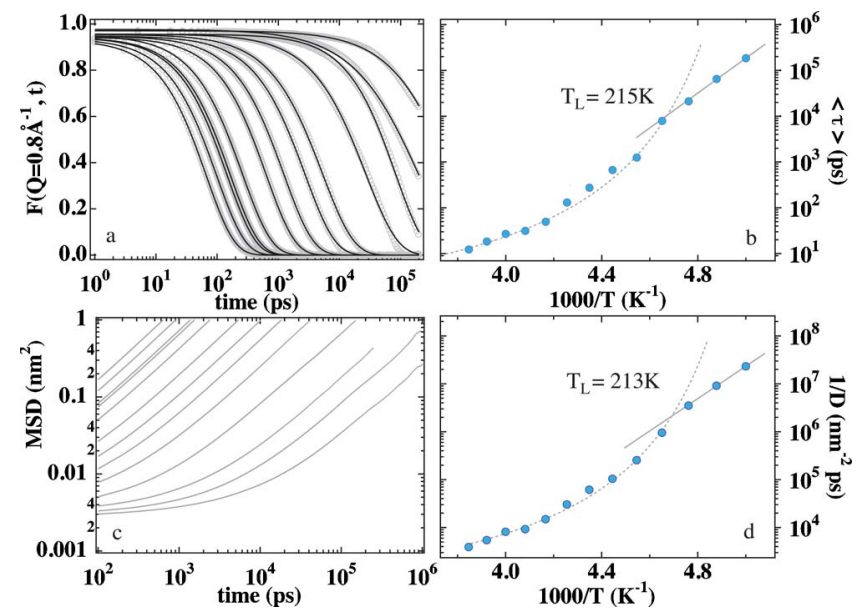

FIG. 3. (Color online) Simulation results. (a) Oxygen SISF at $Q=0.8 \AA^{-1}$ for several temperatures (from 260 to $200 \mathrm{~K}$, every 5 $\mathrm{K})$. Continuous lines are the best fittings with RCM. (b) Arrhenius plot of the translational relaxation time. The dashed line is the fitting of the Vogel-Fulcher-Tammann (VFT) law and the solid line is the Arrhenius law. (c) Long-time mean-square displacement. (d) Arrhenius plot of the self-diffusion constant. 
lated from the trajectory (Fig. 4, lower panel). The self-part of this quantity is defined as [32]

$$
\chi_{4}(Q, t)=\left\langle\left[F_{l}(Q, t)-\left\langle F_{l}(Q, t)\right\rangle\right]^{2}\right\rangle,
$$

where the subscript $l$ indicates the function is a sum over all particles. Since $\chi_{4}(Q, t)$ is related to spontaneous fluctuations, its direct measurement is very difficult. Much easier way is the numerical computation.

The general features of $\chi_{4}(Q, t)$ for bulk water resemble the ones for Lennard-Jones systems [32]. The power-law dependences of the short-time regime and the growth of the peak height of $\chi_{4}(Q, t)$ as one approaches $T_{L}$ are evident. Comparing the two panels of Fig. 4, one notices that while the $\chi_{T}(Q, t)$ peak height has a maximum at $T=T_{L}$, $\chi_{4}(Q, t)$ peak height keeps increasing even below $T_{L}$. This phenomenon may be understood by considering that these two quantities are related by the inequality [16] $\chi_{4}(Q, t) \geq\left(k_{B} / c_{p}\right) T^{2} \chi_{T}^{2}(Q, t)$. This inequality implies that since the specific heat of confined water has a peak at the dynamic crossover temperature $[5,12]$, the dynamic response function $\chi_{T}(Q, t)$ may decrease after $T_{L}$ to keep $\chi_{4}(Q, t)$ growing.

In conclusion, we showed that bulk water simulations are able to reproduce our experimental findings of the threedimensional confined water. The maximum of $\chi_{T}^{*}(Q)$ happens at the dynamic crossover temperature $T_{L}$ and it is not originated from the confinement. On the other hand, the peak height of $\chi_{4}(Q, t)$, which is a measure of the dynamic heterogeneity, continues to increase below $T_{L}$.

Research at MIT is supported by the Department of Energy under Contract No. DE-FG02-90ER45429 and at University of Florence by MIUR and CSGI. SNS in ORNL is sponsored by the Scientific User Facilities Division, Office
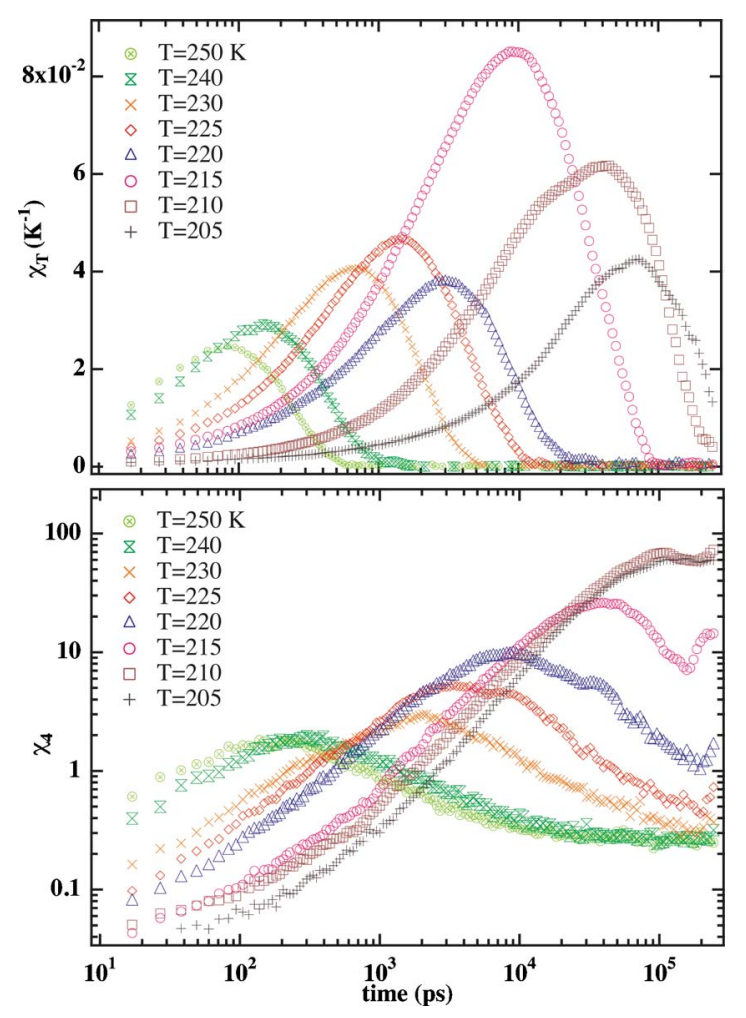

FIG. 4. (Color online) Upper panel: Dynamic response function $\chi_{T}(Q, t)$ for TIP4P-Ew water at several temperatures for $Q=1.0 \AA^{-1}$. Lower panel: $\log -\log$ plot of $\chi_{4}(Q, t)$ for $Q=1.0 \AA^{-1}$.

of Basic Energy Sciences, U.S. Department of Energy. Dry cement powder (CALUSCO) was obtained by CTGItalcementi Group, as a generous gift.
[1] N. K. Gilra, Phys. Lett. 28A, 51 (1968).

[2] C. A. Angell, Annu. Rev. Phys. Chem. 34, 593 (1983).

[3] A. Faraone et al., J. Chem. Phys. 121, 10843 (2004).

[4] S.-H. Chen et al., Proc. Natl. Acad. Sci. U.S.A. 103, 9012 (2006).

[5] Y. Zhang et al., J. Phys.: Condens. Matter 20, 502101 (2008).

[6] K. Ito, C. T. Moynihan, and C. A. Angell, Nature (London) 398, 492 (1999).

[7] H. Tanaka, Europhys. Lett. 50, 340 (2000).

[8] E. G. Ponyatovsky et al., J. Chem. Phys. 109, 2413 (1998).

[9] F. Mallamace et al., J. Chem. Phys. 127, 045104 (2007).

[10] L.-M. Xu et al., Proc. Natl. Acad. Sci. U.S.A. 102, 16558 (2005).

[11] P. Kumar et al., Phys. Rev. Lett. 97, 177802 (2006).

[12] M. Oguni et al., Chem. Asian J. 2, 514 (2007).

[13] D. Liu et al., Proc. Natl. Acad. Sci. U.S.A. 104, 9570 (2007).

[14] F. Mallamace et al., Proc. Natl. Acad. Sci. U.S.A. 104, 18387 (2007).

[15] M. Lagi et al., J. Phys. Chem. B 112, 1571 (2008).

[16] L. Berthier et al., Science 310, 1797 (2005).

[17] L. Berthier et al., J. Chem. Phys. 126, 184503 (2007).

[18] H. M. Jennings, Cem. Concr. Res. 38, 275 (2008).
[19] F. Ridi et al., J. Phys. Chem. B 113, 3080 (2009).

[20] S. H. Chen, C. Liao, F. Sciortino, P. Gallo, and P. Tartaglia, Phys. Rev. E 59, 6708 (1999).

[21] L. Liu et al., J. Phys.: Condens. Matter 16, S5403 (2004).

[22] M. Vogel, Phys. Rev. Lett. 101, 225701 (2008).

[23] S. Nomura et al., Biopolymers 16, 231 (1977).

[24] V. Samouillan et al., Biomacromolecules 5, 958 (2004).

[25] S. Pawlus, S. Khodadadi, and A. P. Sokolov, Phys. Rev. Lett. 100, 108103 (2008).

[26] J. Swenson et al., J. Phys.: Condens. Matter 19, 205109 (2007).

[27] A. Faraone et al., J. Chem. Phys. (to be published).

[28] H. W. Horn et al., J. Chem. Phys. 120, 9665 (2004).

[29] P. Kumar, G. Franzese, and H. E. Stanley, Phys. Rev. Lett. 100, 105701 (2008).

[30] F. Sciortino, P. Gallo, P. Tartaglia, and S. H. Chen, Phys. Rev. E 54, 6331 (1996).

[31] V. N. Novikov and A. P. Sokolov, Phys. Rev. E 67, 031507 (2003).

[32] C. Toninelli, M. Wyart, L. Berthier, G. Biroli, and J. P. Bouchaud, Phys. Rev. E 71, 041505 (2005). 